\title{
Health Problems of Ebola Virus Disease Survivours in Sierra Leone, 2015: Making a Case for Follow-Up Care After Discharge from Ebola Treatment Centres
}

\author{
Edmund Presiror Nabena ${ }^{1,2}$, Waheed Ariyo Bakare ${ }^{2,3}$, Olayinka Stephen Ilesanmi ${ }^{*}, 2,4$, \\ Roosevelt Anyanwu ${ }^{2,5}$ Jude Chimaobina Akuechiama, ${ }^{2,6}$, Samuel E. J. Inyang ${ }^{2,3}$ and \\ Temitope Famuyide ${ }^{2}$
}

${ }^{I}$ Braithwaite Memorial Specialist Hospital, Port Harcourt, Rivers State, Nigeria

${ }^{2}$ Africa Union Support to Ebola Outbreak in West Africa, Sierra Leone

${ }^{3}$ Nigeria Centre for Disease Control, Abuja, Nigeria

${ }^{4}$ Department of Community Health, Federal Medical Centre, Owo, Ondo State, Nigeria

${ }^{5}$ WHO Afro Lab for Viral Hemorrhagic Fever, Virology Unit Central Research Laboratory, College of Medicine, University of Lagos, Nigeria

${ }^{6}$ Swift Emergency Response International, Lagos, Nigeria

\begin{abstract}
Background: The number of the survivours of the Ebola Virus Disease (EVD) is increasing. They have reported health problems.

Objective: This study aimed to document the health problems of EVD survivour in Bombali District Sierra Leone.

Methods: This was a cross sectional study conducted at Bombali District, Sierra Leone in February, 2015. Participants were EVD survivours admitted and discharged from September, 2014 to February 2015. In all, 304 survivours participated in the study by filling an interviewer administered questionnaire. Data were analyzed with SPSS version 21.0 using descriptive statistics. Association between independent variables was explored with chi square test. The level of statistical significance was set at $\mathrm{p}<0.05$.
\end{abstract}

Results: The median age of survivour was 24 years (range 1-89 years), 105 (34.5\%) were $<18$ years, and more than half of the total responders $(60.9 \%)$ were females, 92 (30.3\%) were discharged in November, 2014. All $189(62 \%)$ had health problem: $65(34 \%)$ and $124(66 \%)$ had single and multiple complaints respectively. Joint pain was reported by $111(58.7 \%)$, generalized body pain $-91(48.1 \%)$, headache $-81(43 \%)$, eye problems $-65(34 \%)$, psychological problems - 63(33\%). Only 2 (1.06\%) survivours have sought for help. Among survivours of 18 years and above, 148(74.4\%) had health problems $\mathrm{p}<0.001$. Health problems were reported by $101(70.1 \%)$ of survivours with no formal education $\mathrm{p}=0.002$. Also, 52(83.9\%) widow(er) had health problems $\mathrm{p}<0.001$.

Conclusion: There is a need for the establishment of survivour's clinic. Provision of specialized care for survivour is encouraged based on their identified needs. This can be done early to avoid complications.

Keywords: Ebola, Ebola survivour's clinic, Ebola treatment unit, health problems, Sierra Leone, Survivour.

\section{INTRODUCTION}

The 2013/2014 Ebola Virus Disease (EVD) outbreak in West Africa started in Guinea and spread to Sierra Leone, Liberia, Nigeria, Mali, and Senegal. The outbreak in West Africa is large and has been difficult to contain. This is with respect to the number of countries and people affected [1]. Stopping the outbreak and its effects require concerted efforts. Sierra Leone has recorded more than $50 \%$ of confirmed cases $(8,590)$ in this outbreak and 3,535 deaths

*Address correspondence to this author at the Department of Community Health, Federal Medical Centre, Owo, Ondo State, Nigeria;

Tel: +2348032121868; E-mail: ileolasteve@yahoo.co.uk
$(01 / 05 / 2015)$ [2]. With the establishment of more Ebola care centers and improvement in supportive care, more survivours of EVD are listed. A survivour is a person who had suffered Ebola as confirmed by a positive Ebola PCR or serology test, went through the Ebola disease management and survived the disease. The survivour status is confirmed when the patient tests negative to an Ebola PCR test after EVD management.

A lot of efforts has been put into reducing case fatality rate of the virus leading to significant rise in the number of Ebola survivours in Sierra Leone. Such efforts include the input of several international partners who built more EVD treatment units, gave laboratory and other support. With the coming of more Ebola isolation centres and laboratory the 
turnaround time of the EVD testing was reduced and early diagnosis and commencement of supportive care was enabled.

The previous high fatality rates of EVD meant that only few survivours were available for studies. These numbers were too few to generate significant concern or encourage significant research interests into Post-Ebola health complications. Therefore, few participants were included in previous studies and few health problems were identified [3, 4]. Many of the survivours have developed several health problems. Some studies have looked at the general need of Ebola survivours [5]. A survivour reported she experienced hair loss, body aches and insomnia [6]. Headache, tiredness, bone and joint pains, ocular deficits, blurred vision, hearing loss, and neurologic abnormalities such as difficulty in swallowing and sleeping have been reported. Survivours also reported more chronic health problems and limitations due to memory loss or confusion $[3,7,8]$.

The magnitude of the present outbreak in West Africa demands a complete understanding of the health effects and future health needs of the survivours. With increasing number of survivours there is need to garner more effort in protecting and assisting them to overcome any medical and psychosocial challenges they might have. Therefore, the aim of this study was to document the health problems of EVD survivour in Bombali District, Sierra Leone and to proffer solutions to their health challenges, especially those individuals that are more liable to have complications.

\section{METHODS}

The study was conducted in Bombali District of Sierra Leone. Bombali is the second largest district in Sierra Leone based on geographical land mass (after Koinadugu District) and the second most populous district in the North, after Port Loko District. The population of Bombali district as of 2010 was 434,319 [9].

A cross sectional study design was used. Survivours in Bombali District were invited to a survivour's conference organized by the District Health Management Team (DHMT) Bombali district, AFRICAN UNION, WHO, UNICEF and other partners. About 310 survivours attended the conference. The survivours conference held in Kamaranka village to favour those outside the city and Makeni city in February, 2015. Informed consent was sought and obtained from the survivours. Altogether, 304 agreed to participate in the study. They were interviewed individually by health workers and clinically oriented psychosocial welfare officers using a standardized questionnaire in English. The questionnaire has two parts, the first part comprised of sociodemographic characteristics while the second was on present state of health of the survivours. Identical questions were asked from all survivours irrespective of tribe, religion or Ebola Treatment Centre (ETC) where patient was managed. Interpreters were used to aid the understanding of those who could not speak English Language. Survivours were asked about their health challenges prior to being an EVD patient, health problems they were experiencing when the study was conducted, the onset of the symptoms and co-morbid challenges.

\section{Data Management}

Questionnaires were checked for omissions and errors after collection and correction was made where necessary. Data was analyzed with SPSS version 21.0 using descriptive statistics. Association between independent variables was explored with chi square test. The level of statistical significance was set at $\mathrm{p}<0.05$.

\section{RESULT}

Table 1 shows the Socio-demographic Characteristics of EVD survivour in Bombali District of Sierra Leone. The median age of survivour was 24 years (range 1-89 years). Survivour below 18 years were 105(34.5\%). Also, 185 $(60.9 \%)$ participants were females. Concerning level of

Table 1. Socio-demographic characteristics of Ebola survivour in Bombali District Sierra Leone, 2015.

\begin{tabular}{|c|c|c|}
\hline Characteristics & Frequency & Percent \\
\hline \multicolumn{3}{|l|}{ Age Group in Years } \\
\hline$<18$ & 105 & 34.5 \\
\hline $18+$ & 199 & 65.5 \\
\hline \multicolumn{3}{|l|}{ Sex } \\
\hline Male & 119 & 39.1 \\
\hline Female & 185 & 60.9 \\
\hline \multicolumn{3}{|l|}{ Level of Education } \\
\hline No formal education & 144 & 47.4 \\
\hline Primary & 73 & 24.0 \\
\hline Secondary & 68 & 22.4 \\
\hline Tertiary & 19 & 6.3 \\
\hline \multicolumn{3}{|l|}{ Marital Status } \\
\hline Single & 92 & 36.7 \\
\hline Married & 97 & 38.6 \\
\hline Widow/widower & 62 & 24.7 \\
\hline \multicolumn{3}{|l|}{ Tribe } \\
\hline Thimne & 246 & 80.9 \\
\hline *Others & 58 & 19.1 \\
\hline \multicolumn{3}{|l|}{ Occupation } \\
\hline No Job & 108 & 35.5 \\
\hline Student & 88 & 28.9 \\
\hline Trading & 24 & 7.9 \\
\hline Government Worker & 18 & 5.9 \\
\hline Artisan & 15 & 4.9 \\
\hline Farming & 40 & 13.2 \\
\hline Health Worker & 11 & 3.6 \\
\hline
\end{tabular}


education, 144(47.4\%) had no formal education and only $19(6.3 \%)$ had tertiary education. Of the respondents, 53(17.4\%) were orphans, 246(80.9\%) respondents were from Thimne tribe. Respondents without job were 108(35.5\%), health workers were $11(3.6 \%)$. Other socio-demographic characteristics are as shown in Table 1. Fig. (1) shows the number of patients discharged and the months of discharge. Discharge was at the peak in November, 2014, 92(30.3\%) were discharged.

Table 2 shows the ETC of treatment and presence of comorbid factors. Among the respondents 146 (48\%) received care at Kailahun ETC. In Magbenteh ETC 104(34.2\%) received care. Co morbid factors like Hypertension, Diabetes, and Peptic ulcer disease was reported by $7(2.3 \%)$. Fig. (2) shows the presence of symptoms post EVD treatment in the survivour. In all $189(62 \%)$ had symptoms while $65(34 \%)$ and $124(66 \%)$ had single and multiple symptoms respectively.

Table 2. ETC of treatment and presence of comorbid factors in Ebola Survivour in Bombali District Sierra Leone, 2015.

\begin{tabular}{|c|c|c|}
\hline Variable & Frequency & Percent \\
\hline \multicolumn{3}{|l|}{ ETC } \\
\hline Kailahun & 146 & 48.0 \\
\hline Magbenteh & 104 & 34.2 \\
\hline Hastings & 18 & 5.9 \\
\hline Kenema & 14 & 4.6 \\
\hline Mateneh & 10 & 3.3 \\
\hline \multicolumn{3}{|l|}{ Co Morbid Factors } \\
\hline No & 297 & 97.7 \\
\hline Yes* & 7 & 2.3 \\
\hline
\end{tabular}

Fig. (3) shows the number of survivour that experienced various symptoms post discharge from the ETC. Joint pain was reported by $111(58.7 \%)$, generalized body pain by 91(48.1\%), headache $81(43 \%)$, eye problems $65(34 \%)$, psychological problems $63(33 \%)$. Other health conditions were reported as shown in Fig. (4). Only $2(1.06 \%)$ have sought help from the hospital.

Table 3 shows the association between sociodemographic characteristics and occurrence of health problems among EVD Survivours in Bombali District Sierra Leone. Among survivour 18 years and above 148(74.4\%) had health problems post discharge from the ETC compared to $41(39 \%)$ less than 18 years, $p<0.001$. Health problems was reported in $101(70.1 \%)$ of survivours with no formal education compared to $16(84.2 \%)$ of those with tertiary level of education $(\mathrm{p}=0.002)$. Concerning marital status $52(83.9 \%)$ widow(er) had health problems compared with 44(47.8\%) singles $(\mathrm{p}<0.001)$. Among the farmers $34(85 \%)$ reported health problems while $71(65.7 \%)$ of those who were not working reported same type of health problems $(\mathrm{p}<0.001)$.

\section{DISCUSSION}

This study was conducted among Ebola survivours in Bombali District, Sierra Leone to document their health problems. Groups that are more liable to have complications were also identified based on their sociodemographic characteristics. Ebola survivours are increasing in number each week as the prognosis of the disease is becoming positive $[10,11]$. However, little or no plans have been put forward by various Governments of the affected countries [12]. Since the beginning of the 2014 EVD outbreak, more than 400 Ebola survivours have been recorded in Sierra Leone's Bombali District. Though the survivours now have increased acceptability in their communities [11]. According to the United Nations Population Fund (UNPF), in the three most affected countries (Guinea, Liberia and Sierra Leone) they are providing emergency reproductive health kits, clean delivery kits, blood transfusion sets and equipment to support safe deliveries and manage complications of Ebola Survivours, however, no documented health facility has been designated for this project to attend to Ebola survivours [13].

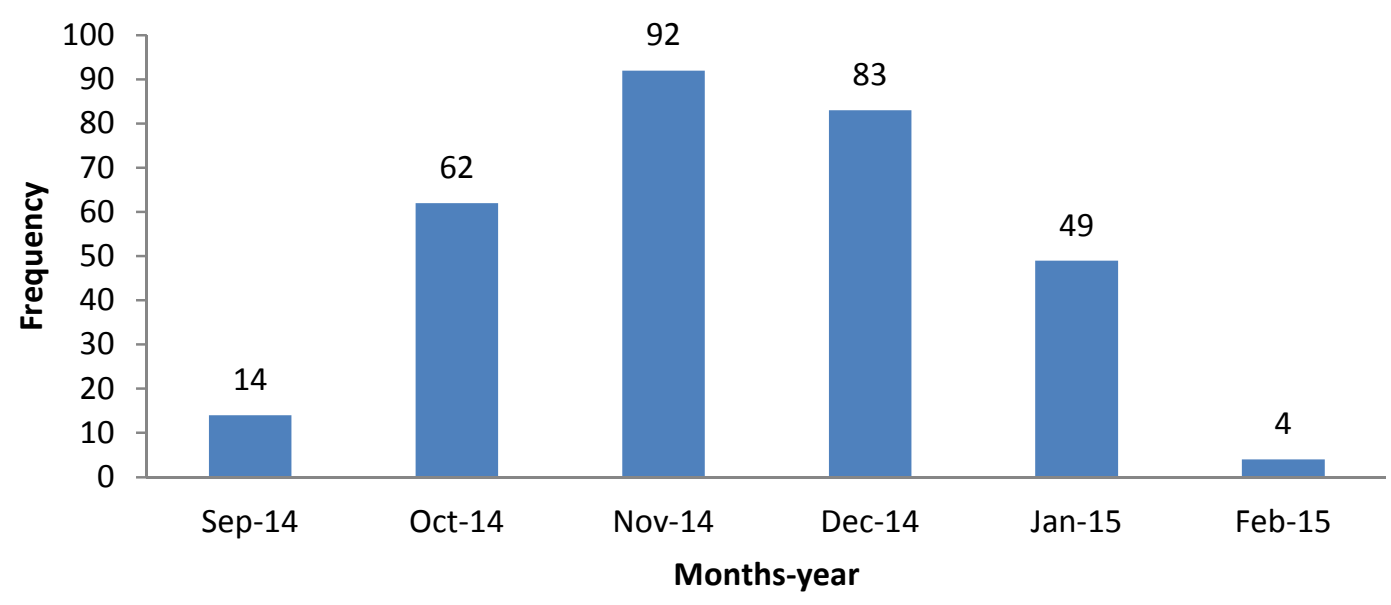

Fig. (1). Number of EVD survivour in Bombali District Sierra Leone, 2015. 


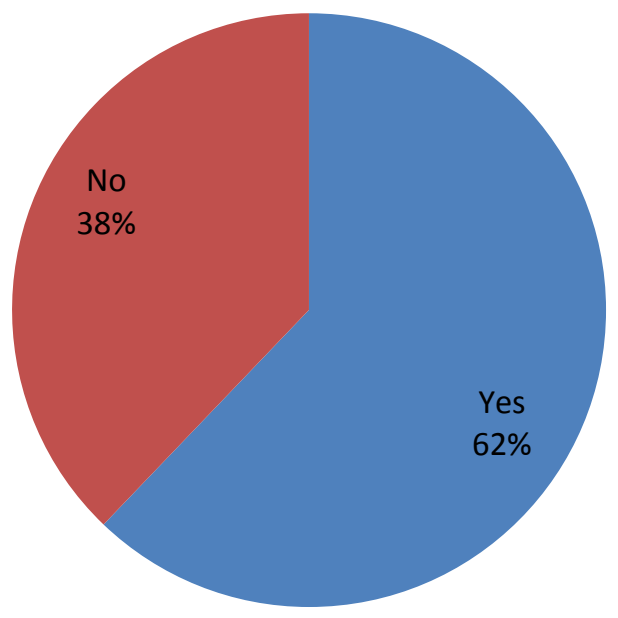

Fig. (2). Presence of symptoms EVD survivour in Bombali District Sierra Leone, 2015.

In our present study more than half of the survivours had health complaints after discharge. About two-third of the survivour with health problems had more than one symptoms. The survivours could not give a detailed report of illnesses prior to EVD because they could barely differentiate between when they started having EVD, malaria or typhoid. More than half had joint pain, a little less than half had general body pains. Other complaints reported were headache, eye complaints, psychological, skin diseases, hearing loss, erectile dysfunction, menstrual irregularity, hair loss, and weight loss. It was noticed that almost all patients who came down with myalgia also developed athralgia, and the severities were different for each survivour. Duration of different complains was also different but most at times it was intermittent and was not really specific to any triggering factor. In another study conducted in Sierra Leone common findings at presentation of Ebola Virus Disease patients included fever, headache, weakness, dizziness, diarrhoea, abdominal pain and vomiting [14]. The findings in survivours differ from what we found after discharge from the ETC.

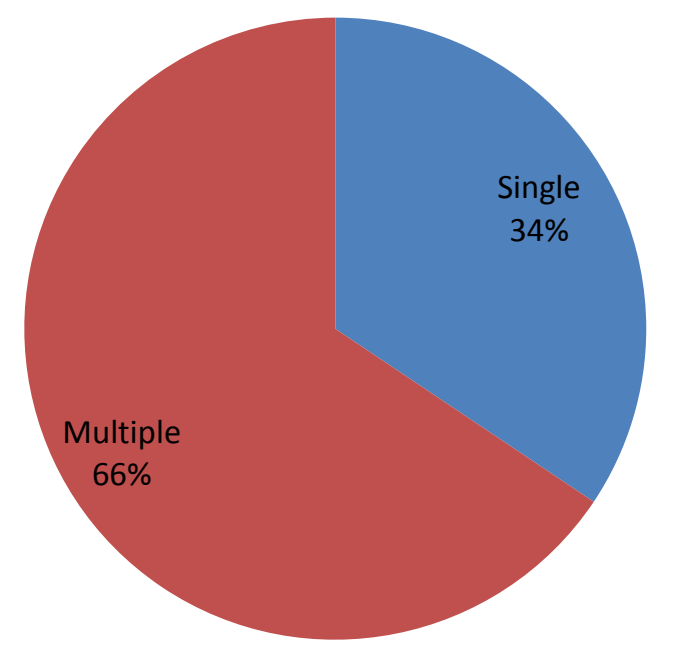

Fig. (3). Number of symptoms in EVD survivour in Bombali District Sierra Leone, 2015.

We noticed that the most frequently occurring symptoms exhibited, had localized organs with special immunity and which were in vacuums and contained fluids, e.g, uveitis (eye ball, vitreous humor enclosed in the eyeball); athralgia (joints, enclosed in a vacuum containing synovial fluid); headache (cranium, intra cranial fluid enclosed in the cranium). Therefore the open question is: whether or not the virus still remains in these tissues even after the blood has tested negative for EVD?

Three out of every four survivours that had health problems were 18 years and above. It is not surprising that

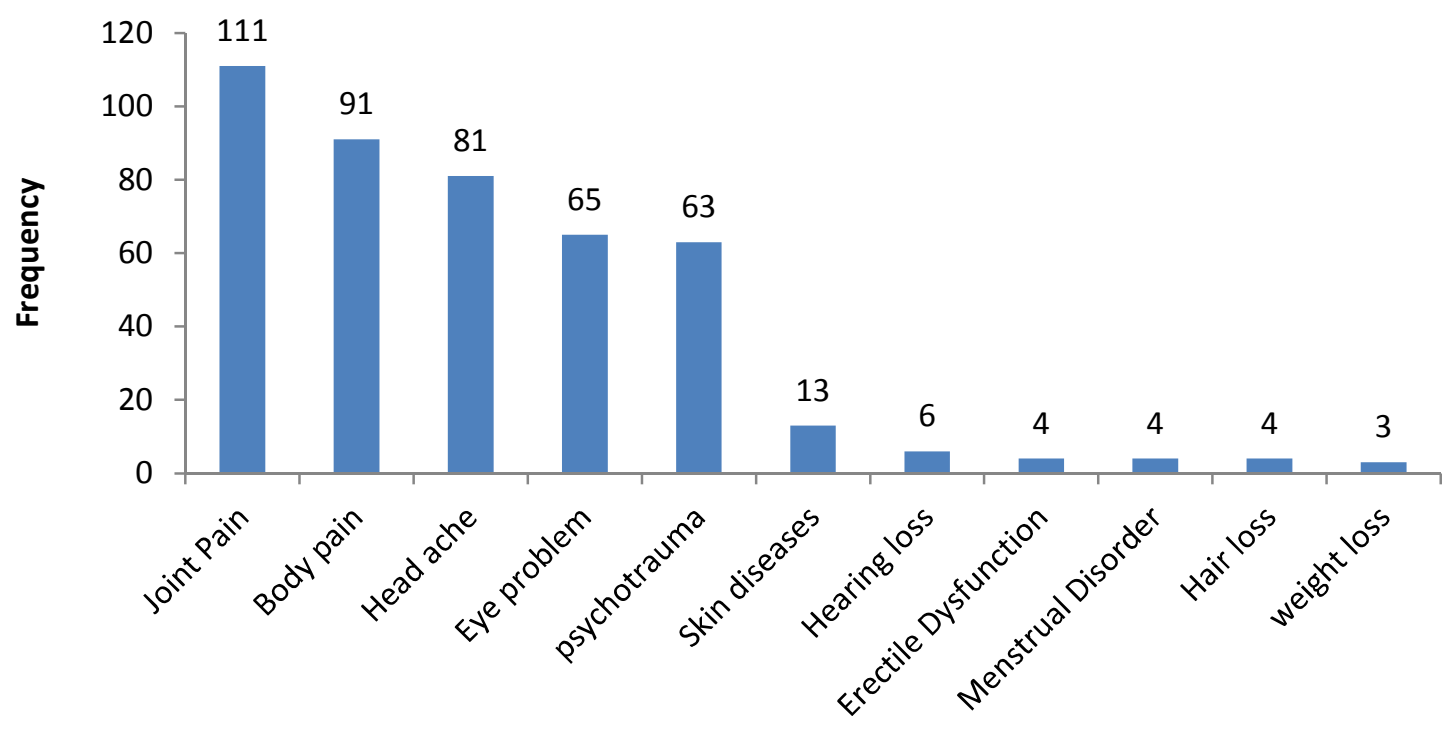

Symptoms

Fig. (4). Proportion of EVD survivour with health problems in Bombali District Sierra Leone, 2015. 
Table 3. Association between socio-demographic characteristics and occurrence of health problems among Ebola survivour in Bombali District Sierra Leone, 2015.

\begin{tabular}{|c|c|c|c|c|}
\hline Variables & \multicolumn{2}{|c|}{ Occurrence of Health Problems } & Chi-Square & p-Value \\
\hline$<18$ & $41(39.0 \%)$ & $64(61.0 \%)$ & 36.47 & $<0.001$ \\
\hline $18+$ & $148(74.4 \%)$ & $51(25.6 \%)$ & & \\
\hline Male & $70(58.8 \%)$ & $49(41.2 \%)$ & 0.932 & 0.334 \\
\hline Female & $119(64.3 \%)$ & $66(35.7 \%)$ & & \\
\hline \multicolumn{5}{|l|}{ Level of Education } \\
\hline No formal education & $101(70.1 \%)$ & $43(29.9 \%)$ & 15.404 & 0.002 \\
\hline \multicolumn{5}{|l|}{ Marital Status } \\
\hline Single & $44(47.8 \%)$ & $48(52.2 \%)$ & 26.394 & $<0.001$ \\
\hline Married & $73(75.3 \%)$ & $24(24.7 \%)$ & & \\
\hline Widow/Widower & $52(83.9 \%)$ & $10(16.1 \%)$ & & \\
\hline \multicolumn{5}{|l|}{ Tribe } \\
\hline Thimne & $148(60.2 \%)$ & $98(39.8 \%)$ & 2.212 & 0.137 \\
\hline *Others & $41(70.7 \%)$ & $17(29.3 \%)$ & & \\
\hline \multicolumn{5}{|l|}{ Occupation } \\
\hline Farming & $34(85.0 \%)$ & $6(15.0 \%)$ & & \\
\hline Health Worker & $9(81.8 \%)$ & $2(18.2 \%)$ & & \\
\hline
\end{tabular}

more adults had health problems. Adults are likely to engage more in energy demanding work that may affect their health. Health problems were reported in a larger proportion of respondents with no formal education. Survivours with no formal education are more often involved in jobs that could affect their health also. Widows (ers) were also more affected compared to their counterparts. Lack or inadequate social support was identified as a known cause of poor health.

As post Ebola Syndrome and other medical complaints emerges from previous outbreaks, it is essential to monitor Ebola survivour's medical complaints in order to assist medical experts to study the relationship between the virus and the complaints the survivours are presenting after discharge. There are still questions about how long survivours are protected from reinfection. It has been difficult to test this outside the Laboratory. There are still no known health facilities designated solely for Ebola survivours in Sierra Leone although several reports acknowledged the existence of some facilities which Ebola survivours visit when they present with one or more symptoms but such facilities also see non Ebola cases as well.

This survey revealed that most Ebola Survivours present with medical complaints within 1 to 4 weeks after discharge from the Ebola Treatment Centers and the proposed Post Ebola Plans by the WHO can only commence after the country is declared Ebola free. Little or no attention is giving to the health of these survivours within the period of discharge from the health facilities to the proposed Post Ebola Phase.

Lack of well-trained health professionals in Sierra Leone and lack of existing Standard Operating Procedures (SOP) and or treatment protocol for specifically the common 
medical complaints of Ebola Survivours may prevent them from visiting the health facilities. Out of pocket payment for health care coupled with the low socio economic status of the survivours are enough to prevent hospital patronage. The poor health care state precedes the outbreak of EVD [15]. Most of the health facilities give blanket treatment which may not be effective to tackle the complaints posed by the survivours.

It is not clearly understood whether post Ebola Syndrome (PES) symptoms pre-existed admission due to poor medical history taking at the point of admission of patient in holding and treatment centres. These make it difficult to know whether these symptoms are attributable to other disease conditions. Therefore detailed history taking at the triage will help. Doctors should be a part of the triage team. Also, further history could be taken at discharge.

\section{RECOMMENDATION}

There is need for the establishment of Survivour clinics to attend to Ebola survivours. A protocol for continuous management of the survivours post discharge is necessary. This can occur in form of post discharge clinical services before the survivour are detached from the health care facilities. This should be done the same way surgeons and clinicians give follow up appointments to their patients. Provision of specialized care for survivour is encouraged based on their identified needs. This can be done early to avoid complications.

The following are also recommended as part of the follow up care, specialists to provide care including otolaryngologist, Physiotherapists, Neurologists, Ophthalmologists, Urologists, Gynecologists, Rheumatologists, Nutritionists and Laboratory Scientists. Basic equipment to be used by the specialist should also be provided. Multi-clinics staffed with relevant specialists in fields of health care delivery to mitigate the symptoms that constitute PES should be established in each district, these specialists would be required to develop standard operating procedures (SOP) for each area of specialization for junior colleagues/clinical officers to adopt. This would avoid haphazard and mere symptomatic treatment.

For a country whose development was largely behind following more than a decade of war, this outbreak could be seen as the proverbial last straw that broke the back of their health system, with many health workers having died from EVD. Intensive training programmes for health personnel are a requirement. Effective health education/communication would help foster early reporting of symptoms from the survivours. This could be through radio, television and fliers in addition to what the survivours received at point of discharge from ETC.

With a large number of survivours from the West African outbreak a large room is created for research into EVD.
Governments need to establish strategic partnership with non-governmental bodies both in the area of treatment and research.

\section{CONFLICT OF INTEREST}

The authors confirm that this article content has no conflict of interest.

\section{ACKNOWLEDGEMENTS}

Entire volunteers of Africa Union Support to Ebola Outbreak in West Africa, (ASEOWA), District Health Management Team (DHMT), Staff of World Health Organization, (WHO) and District Ebola Response Centre (DERC), Bombali District, Sierra Leone.

\section{REFERENCES}

[1] Chan M. Ebola virus disease in west africa - no early end to the outbreak. N Engl J Med 2014; 371(13): 1183-5.

[2] NERC. National Ebola Response Centre (NERC) 2015. (cited 01/05/2015) Available from: http://www.nerc.sl/

[3] Clark DV, Kibuuka H, Millard M, et al. Long-term sequelae after Ebola virus disease in Bundibugyo, Uganda: A retrospective cohort study. Lancet Infect Dis 2015; 15(8): 905-12.

[4] De Roo A, Ado B, Rose B, Guimard Y, Fonck K, Colebunders R. Survey among survivors of the 1995 Ebola epidemic in Kikwit, Democratic Republic of Congo: Their feelings and experiences. Trop Med Int Health 1998; 3(11): 883-5.

[5] Health Day. Ebola Survivors Face Critical Problems 2014. (cited 22/05/2015) Available from: http://consumer.healthday.com/public -health-information-30/centers-for-disease-control-news-120/ebolasurvivors-have-problems-reintgrating-694647.html

[6] Rettner R. What are the long-term effects of Ebola? 2014. [Epub ahead of print]. Available from: http://www.livescience.com/50039ebola-survivors-health-problems.html

[7] U.S. Military HIV Research Program. Study examines long-term adverse health effects of Ebola survivors (Acccessed 21/05/2015) 2015. Available from: http://medicalxpress.com/news/2015-04long-term-adverse-health-effects-ebola.html.

[8] Bausch DG. Sequelae after Ebola virus disease: even when it's over it's not over. Lancet Infect Dis 2015; 15(8): 865-6.

[9] Beamplace.com. Bombali District (accessed 21/05/2015) 2015. Available from: http://www.placebeam.com/?r=poi\&l=9.28333\&g $=-12.38333 \&$ type $=$ all $\&$ title $=$ Laminaia $\% 2 \mathrm{C} \% 20$ Northern $\% 20$ Provi nce $\% 2 \mathrm{C} \% 20$ Sierra $\% 20$ Leone

[10] Welcome to Ministry of Health and Sanitation The Republic of Sierra Leone. 2015. (cited 29/04/2015) Available from: http://health.gov.sl/

[11] UNICEF. At a glance: Sierra Leone, In Sierra Leone, Ebola survivors begin to find acceptance (accessed 29/04/2015) Available from: http://www.unicef.org/infobycountry/sierraleone_80212.html

[12] Gostin LO, Friedman EA. Ebola: A crisis in global health leadership. Lancet 2014; 384(9951): 1323-5.

[13] UNFPA. News on News on Ebola 2015. (accessed 29/04/2015) Available from: http://www.unfpa.org/emergencies/ebola-outbreak

[14] Schieffelin JS, Shaffer JG, Goba A, et al. Clinical illness and outcomes in patients with ebola in Sierra Leone. N Engl J Med 2014; 371(22): 2092-100.

[15] Pieterse P, Lodge T. When free healthcare is not free. Corruption and mistrust in Sierra Leone's primary healthcare system immediately prior to the Ebola outbreak. Int Health 2015; pii: ihv024. 